\title{
Resource And Traffic Based Congestion Control(Rtcc) In Wsn
}

\author{
V. Eswari, K. Marimuthu, K. Bergin Shyni \\ Assistant Professor ${ }^{1,2,3}$ \\ Department of Information Technology ${ }^{1,2}$ \\ Department of Computer Science and Engineering ${ }^{3}$ \\ P.A College of Engineering and Technology, Pollachi, India ${ }^{1,2}$ \\ Dr. Sivanthi Aditanar College of Engineering, Tiruchendur, India ${ }^{3}$
}

\begin{abstract}
To afford the congestion control design for wireless sensor network using RTCC (Resource and Traffic based Congestion Control) conceive. As the number of node increases in the wireless sensor network, the facts and figures developed by such node also is increased this directs to jamming. There are numerous sources for jamming. They are buffer overflow, coexisting transmission, package collision and numerous to one environment. Jamming determinants package loss, which in turn decreases throughput and power effectiveness. Therefore congestion in WSN's desires to be controlled for high energy-efficiency, to extend scheme lifetime, improve Quality of Service (QoS) in terms of throughput and package decrease ratio along with the packet delay. This design will supply the blended means of both asset and traffic congestion control based on the amount of mesh traffic utilizing duty-cycle adjustment of sensor nodes for wireless sensor networks. This RTCC design should advance the mesh concert of the wireless sensor network.
\end{abstract}

Keywords: - Congestion control Scheme, Duty-Cycle parameter, Resource control approach and Traffic control approach. Wireless Sensor Network, QoS.

\section{INTRODUCTION}

A Wireless Sensor Network (WSN) is a distributed scheme comprised of large number of little batterypowered apparatus that sense and assembles information about the natural environment. It is a budding expertise that has a spacious range of promise submissions encompassing natural environment supervising, smart spaces, medical systems, and robotic traveling round fundamentally, each sensor node comprises of feeling, processing, transmission, mobilizer, place finding and power units.

The figure 1 displays the rudimentary architecture of a WSN. Sensor nodes are usually speckled in a sensor area, which is an area where the sensor nodes are established. A sensor node coordinates amidst them to make high-quality information about the corporeal natural environment. Each of these dotted sensor nodes has the capability to assemble and plans facts and figures either to other sensors or back to an external groundwork station. A base-station may be repaired node or a wireless node competent of connecting the sensor mesh to an existing communications infrastructure or to the internet where a user can have access to the reported facts and figures.

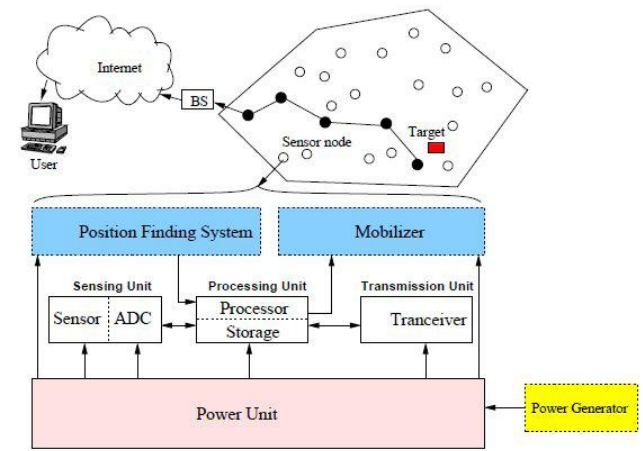

Figure 1. Basic architecture of Wireless Sensor Network

There are two locations where the jamming will happen in WSN, they are node grade and connection grade jamming. In node-grade congestion it is initiated by safeguard overflow in the node and can outcome in package decrease, and augmented lining up delay. Link-level jamming will rises the bundle service time, and decreases both connection utilization and general throughput, and wastes power at the sensor nodes. Both congestions have direct influence on power. 
The jamming control advances can be generalized into two classes; they are resource control and traffic command. When jamming happens, resource control tries to boost resource either by expanding the reception capability of the node or activating the inactive nodes. The traffic command approach is accomplished by adjusting traffic rate at source nodes or intermediate nodes.

Congestion control should be implemented at the MAC (Medium Access Control) layer, rather than at an upper layer such as the transport layer. RTCC scheme is applied over the MAC level in alignment to avoid difficulty of the conceive. In RTCC, the congestion command problem is localized to the MAC level (Data-Link level) duty-cycle parameter. The significant attributes of MAC protocols are collision avoidance, energy good organization scalability in node density, latency, and fairness, throughput and bandwidth utilization. Jamming command has been advised as a crucial, because the node will start to hold up or fall of packets, which will decrease the throughput. Second jamming will increase the power dissipation rate of sensor node reduces the network life span. Happening ally hinders the equitable happening detection and reliable facts and figures transmissions. The asset control approach is achieved by expanding the receiving capability of the receiving node and activating more nodes that are near to the congested node in sleep throughout the jamming. The traffic control approach avoids the jamming by reducing the incoming rate of the package of the parent node as the accessible resource.

\section{RELATED WORK}

The ADCC design, a duty-cycle based jamming organizes design for wireless sensor systems. This can reduce the frequency of changing the transmission rate and can decrease the control package transparency by increasing the package answer rate of the obtaining node and decreasing the packet transmission rate of the sending node. ADCC has the advantage of preventing jamming and improving reliability [1]. The event-driven environment of wireless sensor systems (WSNs) directs to unpredictable mesh load. As a result, jamming may occur at sensors that obtain more facts and figures than they can ahead, which causes energy waste, throughput reduction, and package loss. A rate-based fairness-aware jamming control (FACC) protocol, which controls jamming and achieves in the region of equitable bandwidth share for distinct flows. In FACC, intermediate relying sensor nodes into near-source nodes and near-sink nodes. Near-source nodes sustain a per-flow state and allocation, near-sink nodes do not need to sustain a per-flow state and use a trivial probabilistic dropping algorithm based on line residence and strike regularity [2]. Reliable Muti Segment Transport (RMST) protocol is a hop-by-hop reliable transport protocol built on top of directed diffusion in which packet loss is recovered by hop using caches in the intermediate nodes. Event-toSink Reliable Transport (ESRT) protocol, the rate allocation is centrally computed at the base station periodically counts the number of received sensor readings and reassign the sensors by broadcasting a new transmission rate. Fusion uses the queue length to measure the level of congestion and integrates three techniques: hop-by-hop flow control, rate control and prioritized MAC. Congestion Detection and Avoidance (CODA) senses both channel and buffer occupancies to measure the congestion level [3].

Resources control approaches such as Topology-Aware Resource Adaptation (TARA) increases the network to alleviate congestion and to improve the throughput. Traffic-Aware Dynamic Routing (TADR) algorithm is proposed to route the packets around the congestion areas and to scatter excessive packets along multiple path consists of idle or under-loaded nodes [3].

The special nature of sensor networks calls for a new approach to alleviating congestion that can suit the application fidelity requirements. Therefore, TARA, a Topology-Aware Resource Adaptation strategy, was planned and its presentation was experimentally evaluated. TARA offers several advantages of topology aware, energy efficient, and distributed. TARA uses a capacity analysis model to determine the needed topology. Detailed recital results show that TARA can achieve data delivery rate and energy consumption that is close to an ideal offline resource control algorithm [3].

The first time attempts to formally define the resource control framework that adjusts the resource provisioning at the hotspot during congestion. In effort to find the optimal resource control under the fidelity and energy constraints, at hand a resource increase and decrease algorithm called EIED (Early Increase/Early Decrease) that tries to adjust the effective channel capacity swiftly to the inward bound traffic volume in an energy-efficient manner, thereby increasing the fidelity(or accuracy) level observed by the application during blocking. EIED algorithm performs better than the calm traffic control scheme in terms of fidelity and energy efficiency that is the key metrics in sensor networks [4].

The B-MAC a carrier sense media access protocol for wireless sensor networks that provides a flexible interface to obtain ultra low power operation, successful collision avoidance, and high channel utilization. To achieve low control operation, B-MAC employs an adaptive prelude sampling scheme to reduce duty-cycle and decrease idle listening. B-MAC supports on-the-fly reconfiguration and provides bidirectional interfaces for system services to optimize performance, whether it is for throughput, latency, or power conservation. Use the model to show the effect of changing B-MAC's parameters and foretell the behavior of sensor network applications [5]. 
The T-MAC a contention-based intermediate Access Control protocol for wireless sensor networks. Applications for these networks have a few individuality (low message rate, insensitivity to latency) that can be exploit an active/sleep duty-cycle. The T-MAC protocol introduces a way of decreasing energy utilization in an explosive atmosphere where the note rate fluctuates, either in time or in location. To touch load variation in time and location T-MAC introduces an adaptive duty cycle in novel way by vigorously ending the energetic ingredient of it. This reduces the sum of energy shattered on idle listening, in which nodes wait for potentially incoming messages, while still maintaining a reasonable throughput [6].

Clogging control usually follows two steps: congestion recognition and congestion control. Precise and efficient congestion revealing plays a crucial role in congestion control in sensor networks. Congestion detection is the slant in which that strangely in the normal interchange is been completed out. That is while a container is been transferred from one node to other quandary events can take place. Congestion is controlled by diverse techniques like blocking detection and forestalling, Event-to-Sink unfailing Transport, Congestion Control and neutrality [7].

\section{DESIGN}

Accessible system there is a combined mechanism for the resource based blockage control in wireless sensor network and does not provide both increasing the reserve as well as activating the uninhabited or sleep nodes during clogging. If duty-cycle is low, then it causes performance filth in terms of latency and throughput. If the obstruction occurs then the performance is degraded all the more.

In the anticipated system, RTCC (Resource and Traffic based Congestion Control) scheme, is a blockage control scheme with duty-cycle fine-tuning for wireless sensor networks. The RTCC system is implemented in excess of a duty-cycle based MAC protocol. It uses in concert a resource control approach, by escalating the packet party rate of the receiving node and alleviating the clogging by activating the redundant or sleep nodes, and traffic control approach, by decreasing the packet transmission rate of the sending node for congestion avoidance. The RTCC scheme is based upon the Duty-Cycle parameters depicted in Figure 2.

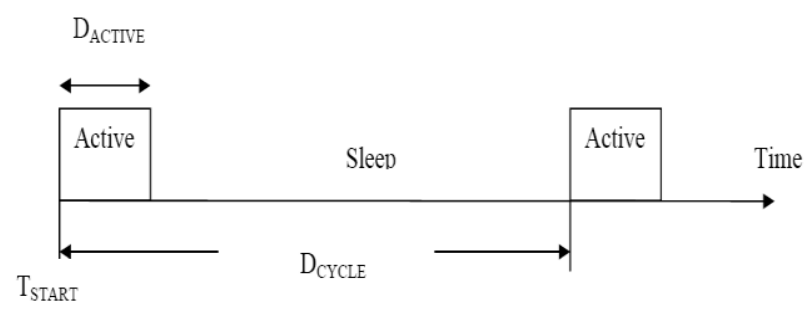

Figure 2. Duty-Cycle parameter

$\mathrm{D}_{\mathrm{ACTIVE}}$ is the extent of the active state in the duty-cycle. $\mathrm{D}_{\mathrm{CYCLE}}$ is the duration between active state to siesta state of the duty-cycle maneuver of node $\mathrm{x} . \mathrm{N}=$ set of child nodes of $\mathrm{x}$. $\mathrm{t}_{\mathrm{INTER} \text { ARRIVAL }}(\mathrm{i})$ is the packet interarrival times of node $i$. $t_{\text {START }}$ is the preparatory point of one duty-cycle. locate the $D_{\text {MIN_ACTIVE }}$ and $D_{\text {MAX_ACTIVE }}$ to the minimum and maximum thresholds of the active duration, in that order, to pledge energy efficiency and the lowest amount packet liberation. The anticipated system for the RTCC scheme has been explained using the subsequent modules.

\section{A. Congestion Detection}

The congestion control in wireless sensor networks is detected from side to side the discrepancy amid the required tune-up time and the duration of the vigorous state in the duty-cycle, which is $\mathrm{D}_{\mathrm{ActivE}}$. The obligatory service time is premeditated by monitoring the packet inter-arrival times of the adolescent nodes by using Equation (1).

$\mathrm{s}(\mathrm{x})=\mathrm{D}_{\mathrm{CYCLE}}(\mathrm{x}) \mathrm{x} \quad \sum_{i=1}^{N} \frac{1}{t \text { INTER-ARRIVAL }(i)}$

Equation (2) shows $\mathrm{d}(\mathrm{x})$ is the congestion degree of the node $\mathrm{x}$. If $\mathrm{d}(\mathrm{x})$ is beneath 0 , network is congested.

$\mathrm{d}(\mathrm{x})=\mathrm{D}_{\mathrm{ACTIVE}}(\mathrm{x})-\mathrm{s}(\mathrm{x})$

To alleviate the congestion, RTCC updates the duration of the active circumstances by considering the calculated congestion extent. The new duration of the active state can be one of three cases as in equation (3). RTCC checks if service time exceeds maximum verge. Then, the new duration of the active state is updated as maximum threshold and RTCC notifies the child nodes of the congestion so that the transmission rate of the child nodes can be abridged. Otherwise, RTCC checks if service time is between minimum and maximum threshold. 
Then RTCC updates active time of the node $\mathrm{x}$ as the calculated service time, which means that RTCC performs resource control. Otherwise if service time is underneath minimum threshold, active time of node $\mathrm{x}$ is updated as minimum threshold to pledge the minimum energy conservation. The new duration of the active state is functional from the next duty-cycle to the next congestion control episode.

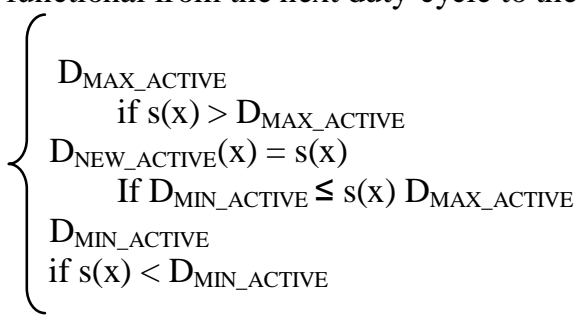

\section{B. Resource Control Approach}

The resource control approach adjusts the resource provisioning at the congested node for alleviating congestion. One of the source control approach is to fiddle with the active time of the duty-cycle of a node. The RTCC proposal adjusts the duration of the active time in the duty-cycle according to the required service time within thresholds. The energy utilization of a node is minimized when the node's effectual channel capacity is equivalent to the cumulative incoming traffic. $R_{i}(t)$ is the effective resource facility of node $i$ at time $t$, and $T_{i}(t)$ is the aggregate incoming traffic volume of node $i$ at time $t$ is given in equation 4.

$\mathrm{R}_{\mathrm{i}}(\mathrm{t})=\mathrm{T}_{\mathrm{i}}(\mathrm{t})$

As in a little while as the congested node detected that its congestion echelon is between the thresholds, it needs to hurriedly establish two vital nodes: the dispenser and the combination. Then, a detour path can be established, starting at the distributor and finale at the unification. As in the distributor, distributes the external traffic between the unique path and the detour path, whereas the merger merges these two flows.

\section{Congestion Notification}

After detecting congestion, congestion control schemes need to circulate the congestion in sequence from the congested node to the upstream sensor nodes that donate to congestion. In the RTCC schemes, when the essential service time exceeds maximum threshold, RTCC notifies the child nodes of the congestion so that the spread rate of the child nodes can be reduced as in figure 3. RTCC uses an unequivocal congestion announcement method by propagation the congestion message to the child nodes.

\begin{tabular}{|l|l|l|l|l|l|}
\hline seq & type & src_id & D_cycle & D_active & change_inter_arrival \\
\hline
\end{tabular}

Figure 3. Congestion Notification format

The variety field contains the message type, and the src_id pasture contains the address of source node. The D_cycle and D_active contain the duty-cycle parameters of the congested node. The change_inter_arrival is the ratio for adjusting the sachet transmission rate of the child nodes. Equation (5) shows the calculation of the new inter arrival value.

Change_inter_arrival $=\frac{s(x)}{\mathrm{D}_{\text {MAX_ACTIVE }}}$

\section{Traffic Control approach}

The node that receives the congestion notification memorandum sets the new transmission rate by multiplying their transmission rates by the revolutionize in bury influx value by using the equation (6).

Interval ${ }_{i}(t)=I_{\text {Interval }}(\mathrm{t}-1) \mathrm{x}$ change_inter_arrival $+\alpha$

The supplementary dwindle of transmission rate for dispensation packets in the queue is $\alpha$. Due to the relative decrease of the transmission rate of child nodes; the traffic control device of RTCC achieves righteousness among the child nodes.

\section{METHODOLOGY}

The RTCC provides the pooled mechanism of the reserve control scheme and traffic control scheme is depicted in figure 4 . The resource control approach, RTCC occasionally calculates the required service time using the incoming packet in sequence of child nodes in the MAC layer. Then it infers whether there is congestion or not through the calculated service time. Subsequently, if the congestion degree is below a convinced threshold, 
the scheme adjusts its duty-cycle to reduce congestion. From the perspective of traffic control loom, if the congestion degree is over the threshold, RTCC notifies the child nodes of the congestion so that the transmission rate of such child nodes can be attuned. Increasing the resources during catastrophe states has been done by using the below ladder.

Resource control approach, as soon as the congestion is detected, in organize to enable more nodes, the two imperative nodes should be situated: distributor and the merger which is depicted in figure 4 . The detour path is reputable between the distributor and the merger. The distributor distributes the incoming traffic between the original path and the detour path, where as merger merges two flows. The above mentioned approach has been implemented using below steps. The resource control approach has been depicted in figure 4 .

\section{E. Traffic Distributor}

Every sensor node keeps trail of the incoming traffic volume from each of its neighbors. Using this information, the congested node can select the upstream neighbor that injects the most packets and send an upstream control packet to that neighbor. If that neighbor node is also bursting at the seams, it repeats the progression until it reaches a node with a low congestion level. That node will then become the traffic dispenser.

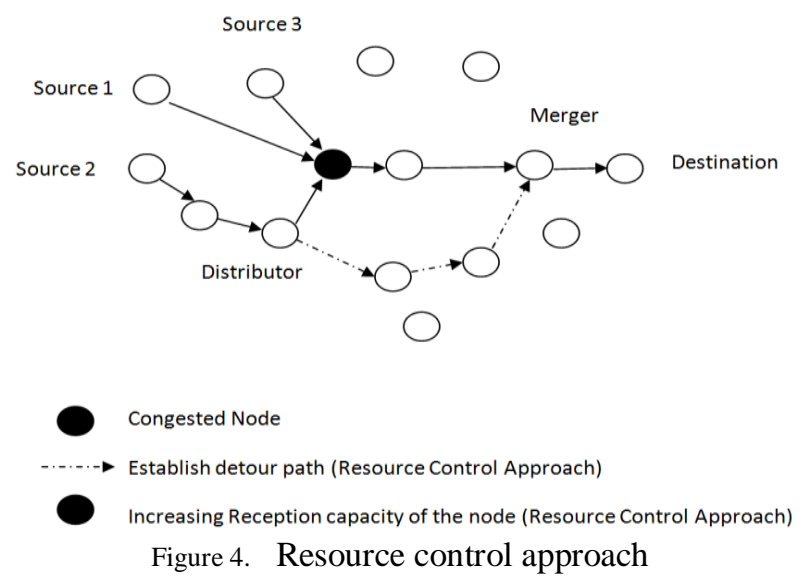

\section{F. Traffic Merger}

To trace a traffic merger, the distributor traces downstream by transport a downstream control packet to the descend that the most traffic is destined for. Therefore, the merger should be located on the steering path from the distributor toward this drop, with a low congestion level. Congestion level should not be the solitary criteria for choosing a merger; a node's location is also essential.

Each node on the routing path keeps trail of the bound count between itself and the sink, which is obtainable from the routing table. As the downstream control sachet reaches a node, which contains the preferred capacity level based on the traffic rate at the distributor, the node can choose whether or not to become a merger based on its distance to the sink. If desired capacity exceeds the resulting competence by this node becoming the merger, then it should just forward the control packet to the next downstream node on the routing path that is closer to the sink. In parallel with the endeavor of tracing the merger, also need to notify the distributor's ID to the merger so that the merger can establish a detour path.

\section{G. Detour Path}

The merger tries to launch the detour path by locally flooding an REQ packet including the Time-ToLive (TTL) field toward the distributor. A node may dispatch manifold REQ packets from a merger due to the temperament of flooding.

When the REQ message reaches the distributor, a contender detour path is time-honored. Usually, the distributor will receive more than one candidate detour path, and it chooses the one whose REQ message has the prevalent TTL value, which corresponds to the smallest path length. To shatter the knot, the REQ message also records the path congestion level, which is the highest congestion level amongst all the nodes on the aspirant detour path, and the distributor chooses a path with a lower obstruction level by conveyance an ACK message toward the merger through the preferred path.

\section{H. Traffic Distribution}

The distributor should rip the gregarious traffic between the original path and the detour path. The distributor checks each packet's destination sink before routing it. Each detour path has a corresponding sink, and if a packet's destination sink does not match the detour path sink, the distributor will only send that packet to the original path. 
V. CONCLUSION

Current exertion focuses on as long as congestion control in wireless sensor networks using RTCC (Resource and Traffic Based Congestion Control), congestion control scheme using duty-cycle amendment for wireless sensor networks in static environment using NS2. This scheme uses both the resource control and traffic control approaches according to the amount of network traffic for the overcrowding evading. Future work focuses on Congestion control in mobile wireless networks using RTCC, an inconsequential congestion control scheme using duty-cycle adjustment.

\section{REFERENCES}

[1] Dongho Lee and Kwangsue Chung, "Adaptive Duty-Cycle Based Congestion Control for Home Automation Networks", IEEE Transaction on Consumer Electronics, vol. 56, issue. 1, pp. 42-47, Feb 2010.

[2] Xiaoyan Yin, Xingshe Zhou, Rongsheng Huang, Yuguang Fang, and Shining Li, "A Fairness-Aware Congestion Control Scheme in Wireless Sensor Networks", IEEE Transactions On Vehicular Technology, vol. 58, No.9 November 2009.

[3] J. Kang, Y. Zhan, and B. Nath, "TARA: Topology-Aware Resource Adaptation to Alleviate Congestion in Sensor Networks", IEEE Transaction on Parallel and Distributed Systems, vol. 18, no. 7, pp. 919-931, July 2007.

[4] J. Kang, Y. Zhang, and B. Nath, "Analysis of Resource Increase and Decrease Algorithm in Wireless Sensor Networks", Proc. of the 11th IEEE Symposium on Computers and Communications (ISCC'06), pp.585-590, June 2006.

[5] J. Polastre, J.Hill, and D.Culler, "Versatile Low Power Media Access for Wireless Sensor Networks", Proc. of the $2^{\text {nd }}$ ACM Conference on Embedded Networked Sensor Systems (SENSYS'04) pp. 95-107, November 2004.

[6] T. Dam and K. Langendoen, "An Adaptive Energy-Efficient MAC Protocol for Wireless Sensor Networks", Proc. of the 1st ACM International Conference on Embedded Networked Sensor Systems (SENSYS'03), pp. 171-180, November 2003.

[7] Rekha Chakravarthi, C.Gomathy, Suraj K. Sebastian, Pushparaj.K, Vinu Binto Mon "A Survey on Congestion control in Wireless Sensor Network", YCSC International Journal of Computer Science \& Communication, Vol.1, No.1, PP.161-164, January-June 2010.

[8] Bret Hull, Kyle Jamieson, Hari Balakrishnan, "Mitigating Congestion in Wireless Sensor Networks", Proc of $2^{\text {nd }}$ ACM Conference on Embedded Networked Sensor Systems (SENSYS'04), November 3-5, 2004.

[9] Ilker Demirkol, Cem Ersoy, and Fatih Alagoz, Bogazici University, "MAC Protocols for Wireless Sensor Networks", IEEE Communication Magazine on Adhoc and Sensor Networks, PP 115-121, April 2006. 